\title{
Does 10-Hz Cathodal Oscillating Current of the Parieto-Occipital Lobe Modulate Target Detection?
}

\author{
Sarah S. Sheldon ${ }^{1 *}$ and Kyle E. Mathewson ${ }^{1,2}$ \\ ${ }^{1}$ Department of Psychology, Faculty of Science, University of Alberta, Edmonton, AB, Canada, ${ }^{2}$ Neuroscience and Mental \\ Health Institute, Faculty of Medicine and Dentistry, University of Alberta, Edmonton, AB, Canada
}

\section{OPEN ACCESS}

Edited by:

Domenica Veniero,

University of Glasgow,

United Kingdom

Reviewed by:

Christian Keitel,

University of Glasgow, United Kingdom

James Dowsett,

Ludwig-Maximilians-Universität

München, Germany

*Correspondence:

Sarah S. Sheldon

ssheldon@ualberta.ca

Specialty section: This article was submitted to

Perception Science,

a section of the journal

Frontiers in Neuroscience

Received: 15 December 2017

Accepted: 01 February 2018

Published: 19 February 2018

Citation:

Sheldon SS and Mathewson KE

(2018) Does 10-Hz Cathodal

Oscillating Current of the

Parieto-Occipital Lobe Modulate

Target Detection?

Front. Neurosci. 12:83.

doi: 10.3389/fnins.2018.00083
The phase of alpha $(8-12 \mathrm{~Hz})$ brain oscillations have been associated with moment to moment changes in visual attention and awareness. Previous work has demonstrated that endogenous oscillations and subsequent behavior can be modulated by oscillating transcranial current stimulation (otCS). The purpose of the current study is to establish the efficacy of cathodal otCS for modulation of the ongoing alpha brain oscillations, allowing for modulation of individual's visual perception. Thirty-six participants performed a target detection with sham and $10-\mathrm{Hz}$ cathodal otCS. Each participant had two practice and two experimental sets composed of three blocks of 128 trials per block. Stimulating electrodes were placed on the participant's head with the anode electrode at $\mathrm{Cz}$ and the cathode electrode at $\mathrm{Oz}$. A $0.5 \mathrm{~mA}$ current was applied every $100 \mathrm{~ms}(10 \mathrm{~Hz}$ frequency) during the otCS condition. The same current and frequency was applied for the first 10-20 s of the sham condition, after which the current was turned off. Target detection rates were compared between the sham and otCS experimental conditions in order to test for effects of otCS phase on target detection. We found no significant difference in target detection rates between the sham and otCS conditions, and discuss potential reasons for the apparent inability of cathodal otCS to effectively modulate visual perception.

Keywords: alpha oscillations, transcranial current stimulation, entrainment, detection, negative findings

\section{INTRODUCTION}

Oscillating neural activity enables the brain to communicate and coordinate across different areas in order to carry out important cognitive functions. Over the last decade, there has been a resurgence of interest in oscillatory activity due to recent technological advances that enable noninvasive modulation of these brain oscillations (Herrmann et al., 2013; Fröhlich, 2015; Fröhlich et al., 2015). In particular, transcranial current stimulation (tCS) has become a popular method because it provides the possibility to modulate the phase, amplitude, and frequency of ongoing oscillatory activity (Paulus, 2011).

The most common applications of tCS involves the delivery of the electrical stimulation as either a direct current (i.e., current of a constant intensity and polarity) or an alternating current (i.e., current that oscillates between negative and positive polarity). Anodal (positive polarity) and cathodal (negative polarity) transcranial direct current stimulation (tDCS) can modulate the neuronal response threshold by inducing depolarization or hyperpolarization, respectively (Jackson et al., 2016; Paulus et al., 2016). On the other hand, transcranial alternating current stimulation (tACS) can modulate ongoing neuronal activity in a frequency-specific manner. It is thought that 
tACS effects response thresholds in a manner similar to tDCS except that alternating between positive (anodal) and negative (cathodal) current results in the neural oscillations becoming entrained to the timing of the alternating current (Antal and Paulus, 2013; Herrmann et al., 2013; Reato et al., 2013; Vosskuhl et al., 2015). This means that tACS can be used to manipulate oscillatory activity in an experimental setting to understand the relevance of such induced oscillations for cognition.

Although numerous studies have demonstrated that these electrical stimulation methods can effect perception (Antal et al., 2004b; Antal and Paulus, 2008; Neuling et al., 2012a; Helfrich et al., 2014) and cognition (Marshall et al., 2006; Zaehle et al., 2011; Antonenko et al., 2016; Simonsmeier and Grabner, 2017), it seems that many other studies have found little to no evidence supporting the efficacy of these techniques (Brignani et al., 2013; Tremblay et al., 2014; Horvath et al., 2015; Marshall et al., 2016; Veniero et al., 2017). Therefore, here we used a well-studied paradigm of alpha oscillations affecting visual perception as a test of the feasibility of using tCS to manipulate oscillations and cognition.

Using tACS with a DC-offset is referred to as oscillating transcranial current stimulation (otCS). This technique can be thought of as a combination of tDCS and tACS, and has been shown to be effective for boosting memory (Marshall et al., 2006) and modulating corticospinal excitability (Jaberzadeh et al., 2014). Despite evidence for the effectiveness of otCS, there are very few studies that have used this method. To further explore this method, we utilize cathodal otCS here to manipulate posterior parietal alpha oscillations and test if there was any influence on target detection.

Our decision to use cathodal otCS comes from converging evidence suggesting that it would be more effective at modulating posterior parietal endogenous brain oscillations than anodal otCS and the more traditional tACS. For example, Marshall et al. (2006) showed that slow-wave anodal otCS boosted endogenous brain oscillations in a frequency-specific manner and facilitated the cognitive function of those oscillations. However, intracranial tACS at the same frequency did not replicate this effect (Lafon et al., 2017). In a study by Alekseichuk et al. (2016) using combined tCS and fMRI, the authors found that anodal tDCS over the occipital cortex during visual perception led to a significant increase in the BOLD activity in the stimulated area, whereas there was no significant BOLD activity change during the $10 \mathrm{~Hz}$ tACS stimulation (though there was a wide spread decrease of the BOLD signal immediately after the stimulation). At this point, it might seem logical to use anodal otCS over cathodal otCS because anodal stimulation induces somatic polarization in neural populations, resulting in increases of firing rates, while opposite effects are induced with cathodal stimulation (Polanía et al., 2018). However, previous studies found that natural alpha power increase is associated with inhibition in the firing rates and smaller BOLD signal (Laufs et al., 2003; Haegens et al., 2011). In addition, cathodal tDCS over the primary visual cortex has been shown to induce longer lasting effects on visual cortical excitability than anodal stimulation (Antal et al., 2004a). Furthermore, using largescale simulations of cortical networks, Ali et al. (2013) found that hyperpolarizing-only oscillating stimulation entrained the cortical network just as effectively as tACS; in comparison, the depolarizing-only stimulation exhibited reduced effects on the ongoing oscillation dynamics compared to tACS. Together, this evidence supports the idea that cathodal otCS may be more effective at modulating posterior parietal endogenous brain oscillations than anodal otCS and tACS.

Brain oscillations within the alpha $(8-12 \mathrm{~Hz})$ frequency band have emerged as a marker of visual perception and selective attention (Klimesch et al., 2007; Mathewson et al., 2011; Klimesch, 2012). We and others have shown that target detection depends on the phase of alpha oscillations at the moment of target onset (Busch et al., 2009; Mathewson et al., 2009; de Graaf et al., 2013), which can be explained as alpha acting as a pulsating inhibition in the brain. We have found using fast optical imaging that these alpha oscillations relevant for detection can be localized to the posterior parietal cortex (Mathewson et al., 2014). Support for this theory comes from studies using EEG and MEG showing rhythmic visual stimulation modulation of alpha oscillations predicts subsequent periodic patterns in target detectability (Mathewson et al., 2014; Spaak et al., 2014; Kizuk and Mathewson, 2017). Furthermore, this phenomenon seems to be due to entrainment of the endogenous alpha oscillation by the rhythmic visual stimuli (Notbohm et al., 2016; Gulbinaite et al., 2017) rather than some aspect of rhythmically presented visual stimulation (for a critical discussion see Keitel et al., 2014).

In comparison to the classical rhythmic sensory stimulation protocols mentioned above which interacts with the entire visual system, the use of tCS offers the advantage of directly stimulating cortical targets (Brignani et al., 2013). The aim of the current study was to provide a proof of principle that the entrainment of ongoing neural oscillations by rhythmic visual stimulation can be replicated with cathodal otCS at the same frequency. The present study aims to address this issue by attempting to control the phase alpha oscillations in the posterior parietal cortex during visual perception.

\section{MATERIALS AND METHODS}

\section{Participants}

Thirty-six participants from the University of Alberta community participated in the study (mean age $=21$; age range $=17-32,10$ males). Participants were all right-handed, and had normal or corrected normal vision and no history of neurological problems. All participants gave informed written consent, were either compensated at a rate of $\$ 10 / \mathrm{h}$ or given research credit for their time, whichever was applicable. The study adhered to the tenets of the Declaration of Helsinki and was approved by the Internal Ethics Board at the University of Alberta.

\section{Target Detection Task}

Participants were seated $57 \mathrm{~cm}$ away from a $1920 \times 1090$ pixel $^{2}$ ViewPixx/EEG LCD monitor (VPixx Technologies, Quebec, Canada) with a refresh rate of $120 \mathrm{~Hz}$, simulating a CRT display with LED backlight rastering. The rastering, along with 8-bit digital TTL output triggers yoked to the onset 
and value of the top left pixel, allowed for submillisecond accuracy in pixel illumination times, which were confirmed with a photocell prior to the experiment. Stimuli were presented on a $50 \%$ gray background using a Windows 7 PC running MATLAB R2012b with the Psychophysics toolbox (Version 3; Brainard, 1997; Pelli, 1997). See Figure 1A for the stimulus dimensions. Video output was sent to the ViewPixx/EEG with an Asus Striker GTX760 (Fremont, CA) graphics processing unit.

Each trial began with a black fixation cross presented at the center of the monitor for $400 \mathrm{~ms}$. The fixation cross was followed by a blank screen. The blank screen remained for 200, 225, 266.66, $283.33,300,341.66,374.99$, or $408.33 \mathrm{~ms}$ (target stimulus onset asynchrony; tSOA) after which the target appeared for $8.33 \mathrm{~ms}$ (one monitor refresh). The target was followed by a backward mask lasting for $8.33 \mathrm{~ms}$ with a constant $41.7 \mathrm{~ms}$ target-mask SOA (mSOA). Following the mask offset, the participant had $1000 \mathrm{~ms}$ to respond before the next trial began. There were 128 trials per block, and three blocks per experimental condition. On $20 \%$ of trials, the target was omitted to assess false alarms. A summary of the task sequence can be seen in Figure 1B.

In the first two conditions, the target luminance value was adjusted throughout the task based on a 3-up/1-down staircasing procedure that was chosen because it targeted a 0.5 target detection rate for each individual (García-Pérez, 1998; Kingdom and Prins, 2016). The target luminance value in the final two conditions remained constant and determined for each participant by taking the average target luminance value across the last two blocks of trials in the second staircasing block.

\section{Electrical Stimulation}

A battery-driven stimulator (Oasis Pro, Mind Alive, Canada) was used to deliver a $10-\mathrm{Hz}$ oscillating cathodal transcranial electrical current via rubber electrodes encased in sponges $(5 \times 4 \mathrm{~cm}$; Oasis Pro, Mind Alive, Canada) and soaked in saline solution. The electrodes were attached to the head underneath an EEG Recording Cap (EASYCAP, Herrsching, Germany) with the cathodal electrode (where the current was applied) at $\mathrm{Oz}$ and the anodal electrode placed at $\mathrm{Cz}$. These positions were chosen for maximal stimulation intensity in the parieto-occipital cortex (Neuling et al., 2012b). The stimulation current had a rounded square waveform that was delivered at a $10-\mathrm{Hz}$ frequency. Each stimulation pulse lasted $2.5 \mathrm{~ms}$. The manufacturers designed their stimulating device to produce currents with a rounded square waveform because they found it to reduce the stinging/burning sensation without loss of effectiveness (Siever, 2014). We confirmed that the stimulator produced a rounded square waveform with an oscilloscope. An example of this rounded square waveform can be seen in Figure 2. The onset of each stimulation pulse was recorded by the amplifier via a customized trigger output added to the Oasis Pro stimulator by the manufacturer with the accuracy confirmed with oscilloscopes prior to the experiment.

A

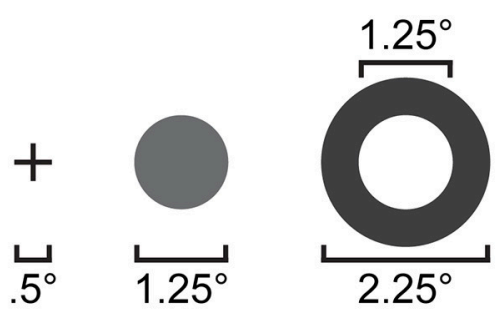

B

Pulse to Target SOA (ms)

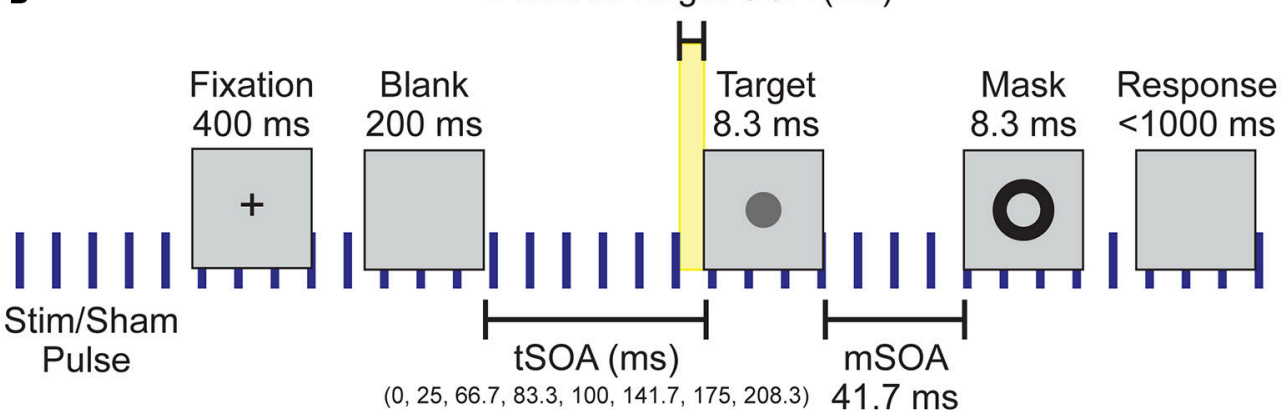

FIGURE 1 | Experimental setup and design. (A) Spatial dimensions of the stimuli, which were presented to subjects at the center of the screen. (B) Individual trial timeline with durations of each screen presentation. Blue vertical lines indicate the continuous application of the $10 \mathrm{~Hz}$ sham or otCS stimulation pulse throughout the task. Highlighted yellow area was the time range between the preceding stimulation (sham or otCS) pulse and the onset of the target which was used to subdivide the trials into $10 \mathrm{~ms}$ bins. 


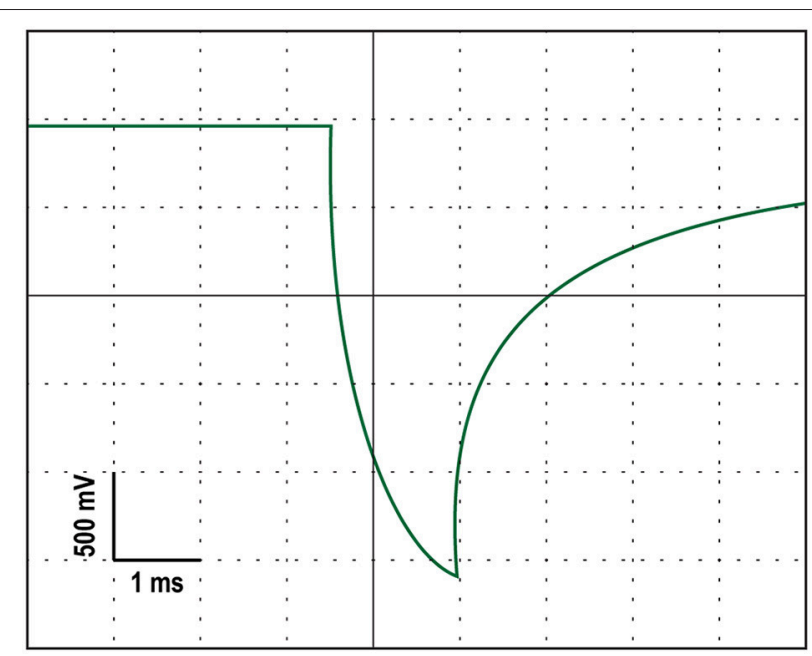

FIGURE 2 | An oscilloscope tracing of the rounded square stimulation waveform produced by the stimulating device.

The intensity of the stimulation current was adjusted for each participant to ensure that they did not experience pain, tingling or other unpleasant sensations. To obtain this threshold, we started with an intensity level of $0.50 \mathrm{~mA}$ (peak-to-peak). We chose to start at this intensity level because participants in the pilot study reported pain at current intensities $0.75 \mathrm{~mA}$. If the participant indicated unpleasant sensations, we decreased the intensity in steps of $0.02 \mathrm{~mA}$ until the participant reported little to no skin sensation. The obtained threshold level ranged between 0.34 and $0.50 \mathrm{~mA}(M=0.46, S D=0.05)$ was used as stimulation intensity in the tCS condition.

The sham condition consisted of a $10 \mathrm{~s}$ fade-in and $20 \mathrm{~s}$ of stimulation at $0.50 \mathrm{~mA}$. The current was then shut off by disconnecting the Oasis Pro stimulator from the stimulating electrodes outsight of the sign of the participant. Disconnecting the stimulating device from the electrodes did not interrupt the stimulation triggers sent to the amplifier, which can therefore be used as control timings. The experimental condition also consisted of a $10 \mathrm{~s}$ fade-in and $20 \mathrm{~s}$ of stimulation at $0.50 \mathrm{~mA}$, after which the current intensity was decreased to the individual's obtained threshold level.

\section{EEG Recording}

During the target detection task, EEG data was recorded using a 16-channel V-amp amplifier (Brain Products, München, Germany) from 15 scalp locations (O1, O2, P7, P3, Pz, P4, P8, T7, C3, Cz, C4, T8, F3, Fz, F4; 10/20 system), a ground electrode at position $\mathrm{Fpz}$, and two reference electrodes, placed at the right and left mastoids, with $\mathrm{Ag} / \mathrm{AgCl}$ sintered ring electrodes (EASYCAP, Herrsching, Germany) in a 20-channel electrode cap (EASYCAP). SuperVisc electrolyte gel and mild abrasion with a blunted syringe tip were used to lower impedances to less than $5 \mathrm{k} \Omega$ for all electrode sites except $\mathrm{Cz}$ which did not have direct contact with the head because it was on top of the stimulating electrode sponge. EEG was recorded online referenced to an electrode attached to the left mastoid. Offline, the data were rereferenced to the arithmetically derived average of the left and right mastoid electrode.

In addition to the 15 EEG sensors, two reference electrodes, and the ground electrode, the vertical and horizontal bipolar EOG was recorded from passive $\mathrm{Ag} / \mathrm{AgCl}$ Easycap disk electrodes affixed above and below the left eye, and $1 \mathrm{~cm}$ lateral from the outer canthus of each eye. Prior to placement of electrodes, the participant's skin was cleaned using Nuprep (an exfoliating cleaning gel) and electrolyte gel was used to lower the impedance of these EOG electrodes to under $5 \mathrm{k} \Omega$ in the same manner as previously mentioned. The bipolar vertical and horizontal EOG was recorded using a pair of BIP2AUX converters in the V-amp auxiliary channels (Brain Products). The EOG electrodes had a separate ground electrode affixed to the central forehead.

Data were digitized at $2000 \mathrm{~Hz}$ with a resolution of 24 bits $(0.049 \mu \mathrm{V}$ steps). Data were collected inside a sound and radio frequency-attenuated chamber (40A-series; ElectroMedical Instruments, Mississauga, Ontario, Canada), with copper mesh covering a window. The lights were left on, and the window was covered during experiments. The only electrical devices inside the chamber were the amplifier, powered from a battery powered laptop located outside the chamber, speakers, keyboard, and mouse, all powered from outside the room, the ViewPixx monitor, powered with DC power from outside the chamber, and a battery-powered intercom. Nothing was plugged into the internal power outlets, and any electrical devices (e.g., cell phones) were removed from the chamber during recording.

\section{Design and Procedure}

For all the participants, the study consisted of one session and took $\sim 90 \mathrm{~min}$. We implemented a single-blind sham-controlled design in which participants underwent two experimental conditions (otCS and sham) in a counterbalanced order. EEG data was simultaneously recorded during both conditions.

The procedure started with the participants performing three practice blocks of the staircased version of the target detection task while the experimenters set-up the EEG cap and electrical stimulation electrodes. After the practice blocks and set-up, the electrical stimulation intensity was determined for each participant using the procedure described above. Next, the participant performed the staircased version of the target detection task a second time under the stimulation sham condition. The average luminance value of the target in the last two blocks of trials was calculated for each participant. Finally, participants performed the target detection task under the otCS and sham experimental conditions (counterbalanced across subjects) using the previously calculated target luminance value.

Although EEG data was recorded throughout the final three conditions, attempts to remove the otCS stimulation artifact with both traditional and advanced multi-step procedures (Liu et al., 2012; Helfrich et al., 2014; Kohli and Casson, 2015) were unsuccessful. This was most likely due to the presence of small fluctuations of stimulation intensity caused by the stimulating device. Therefore, we were not able to examine possible psychophysiological effects. 


\section{Questionnaire}

To obtain possible adverse effects for otCS, a version of a questionnaire introduced by Brunoni et al. (2011) was used. The following side-effects were inquired: headache, neck pain, scalp pain, tingling, itching, burning sensation, skin redness, sleepiness, trouble concentrating, and acute mood change. Participants were asked to indicate the intensity of the side-effect (1, absent; 2, mild; 3, moderate; 4 , severe) and if they attributed the side-effect to the tACS (1, none; 2, remote; 3 , possible; 4 , probable; 5 , definite).

The most reported adverse effects (intensities rated higher than 1) after the experiment were trouble concentrating (70.0\%), sleepiness (66.7\%), and scalp tingling (56.7\%). Ratings for intensity of adverse effects were generally relatively low, except for sleepiness $(M=2.12)$ and trouble concentrating $(M=2.10)$. For the ratings on whether subjects attributed the adverse effects to the stimulation, only tingling achieved an average score above $2(M=2.20)$.

\section{Data Analyses}

Data analysis was performed using MATLAB R2017a (The MathWorks Inc, Natick, MA, USA) and EEGLAB 13.6.5b (Delorme and Makeig, 2004). Statistical analyses were conducted using SPSS 11.5.0 (Chicago, IL) and R 3.3.1 (R Core Team, 2013). To quantify the strength of evidence in the parametric statistical tests, the Bayes factor (BF) was calculated with JASP software (JASP Team, 2018) using the default Cauchy prior width of 0.707 (Jarosz and Wiley, 2014; Wagenmakers et al., 2017). For nonparametric tests, a Bayesian version of the Wilcoxon signed-rank test using a Dirichle process based prior was used to further test the null results (Benavoli et al., 2014).

\section{Target Detection Performance}

First, the trials from the non-staircased version of the target detection task were subdivided into $10 \mathrm{~ms}$ bins based on the time between the preceding stimulation pulse and the onset of the target (pulse to target SOA; see Figure 1B). This was our main independent variable, since we predict that if alpha oscillations are being entrained by the electrical stimulation their phase should influence detection. Because a stimulation pulse was every $100 \mathrm{~ms}$, this meant that there was a total of10 bins. Target detection rates (proportion of targets participants detected) of each participant was calculated for these ten $10 \mathrm{~ms}$ bins after excluding catch trials (where no target appeared) and trials without a valid response. These calculations were performed separately for each stimulation condition (otCS and sham). A test of the mean detection rates across bins between otCS and sham conditions was conducted using a mixed ANOVA where the $10 \mathrm{~ms}$ bins and stimulation condition were within-subject factors, condition order (otCS before sham or sham before otCS) was a between-subject factor, and the participants were treated as a random variable. The ANOVA was performed in $\mathrm{R}$ using the built-in aov function and the ezANOVA function from the ez package (Lawrence, 2016). The analysis yielded a significant interaction between stimulation condition and order of conditions indicating the presence of a sequence effect (see Results section and Figure 4A). The sequence effect was not relevant to the hypothesis that target detection rates will vary in a sinusoidal manner relative to otCS stimulation pulses but not the sham pulses. Therefore, the target detection rates were normalized for each participant in each condition separately and then re-tested with the mixed ANOVA.

Finally, the behavioral data was subdivided into 12 bins of 32 consecutive trials across the three blocks of each stimulation condition and submitted to a repeated-measures ANOVA. This was done to investigate whether there was a change in target detection rates across the condition, since if alpha power increases with stimulation time target detection should get worse.

\section{Sinusoidal Model of Detection Rates}

For each participant and stimulation condition, the sinusoidal function

$$
x(t)=\alpha_{0}+\alpha_{1} \sin (\omega t+\phi)
$$

with intercept $\alpha_{0}$, amplitude $\alpha_{1}$, and phase $\phi$ was estimated for the standardized target detection rates of the ten 10 -ms bins in each stimulation condition. The routine to fit the parameters was initialized with random start values, and used a non-linear leastsquares method. The parameters were limited by the following constraints: $\phi \in(-\pi, \pi) ; \alpha_{1} \in(0, \infty)$; and, frequency $\omega$ was fixed at 0.06 bins/cycle $(100 \mathrm{~Hz})$. To compare the influence of the otCS and sham stimulation pulses on target detection rates, a paired Student's $t$-test was performed on the estimated amplitude $\left(\alpha_{1}\right)$ and a Wilcoxon signed-ranks test was performed on the goodness-of-fit measure adjusted r-square $\left(R_{a d j}^{2}\right)$.

\section{EEG Data}

The average voltage in the $300 \mathrm{~ms}$ baseline prior to the target was subtracted on each trial for every electrode. Trials with absolute voltage fluctuations on any channel greater than $1000 \mu \mathrm{V}$ were discarded, and data was segmented into $1800 \mathrm{~ms}$ epochs aligned to target onset ( $-800 \mathrm{~ms}$ pre-target onset to $1000 \mathrm{~ms}$ post-target onset). Eye movements were then corrected with a regressionbased procedure developed by Gratton et al. (1983). After a second baseline subtraction with $300 \mathrm{~ms}$ pre-target, trials with remaining absolute voltage fluctuations on any channel greater than $500 \mu \mathrm{V}$ were removed from further analysis.

\section{RESULTS}

The mixed ANOVA on the mean detection data yielded no significant main effects or interactions (Figure 3A) except for the interaction between stimulation condition and stimulation condition order $\left[F_{(1,646)}=38.20, p<0.001\right]$. This indicates that there was a sequence effect in that mean target detection rates were greater in the second stimulation condition compared to the first, regardless of whether sham came before otCS (sham condition: $M=0.46, S E=0.05$; otCS condition: $M=0.51$, $S E=0.04$ ) or otCS came before sham (sham condition: $M=0.49$, $S E=0.05$; otCS condition: $M=0.45, S E=0.04$ ). All other main effects and interactions had an $F$-value of less than 1 .

A Bayesian mixed ANOVA suggests that the data were 445341.97:1 in favor of a model containing stimulation 


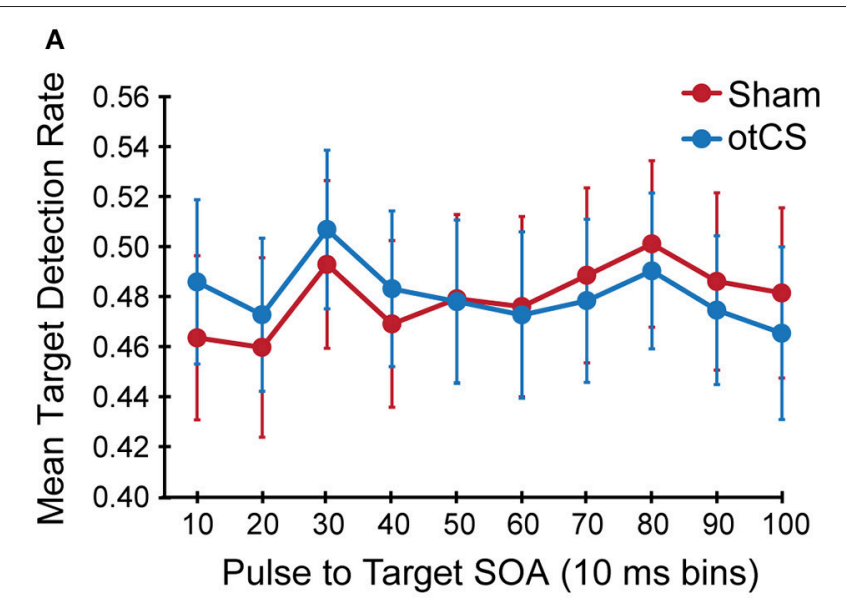

\section{B}

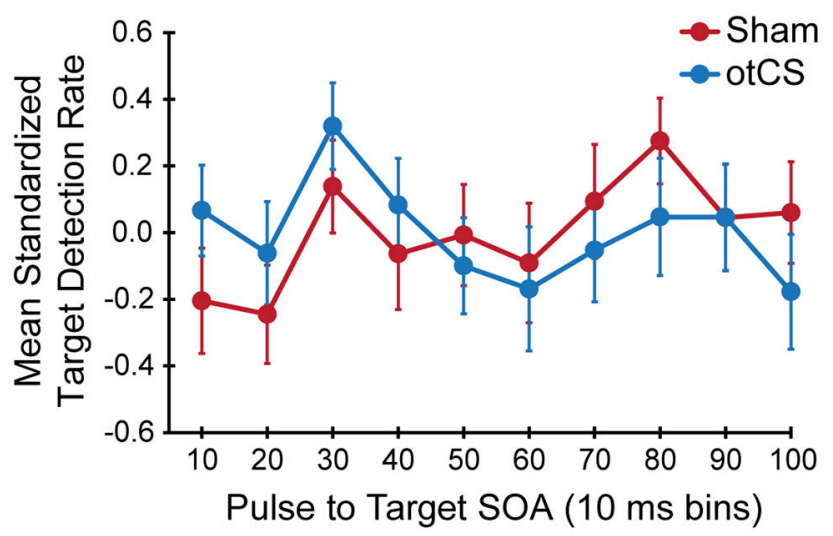

FIGURE 3 | (A) Mean target detection rates and (B) mean standardized target detection rate in each $10 \mathrm{~ms}$ bin during the sham and otCS stimulation conditions. Error bars indicate the standard error (SE).

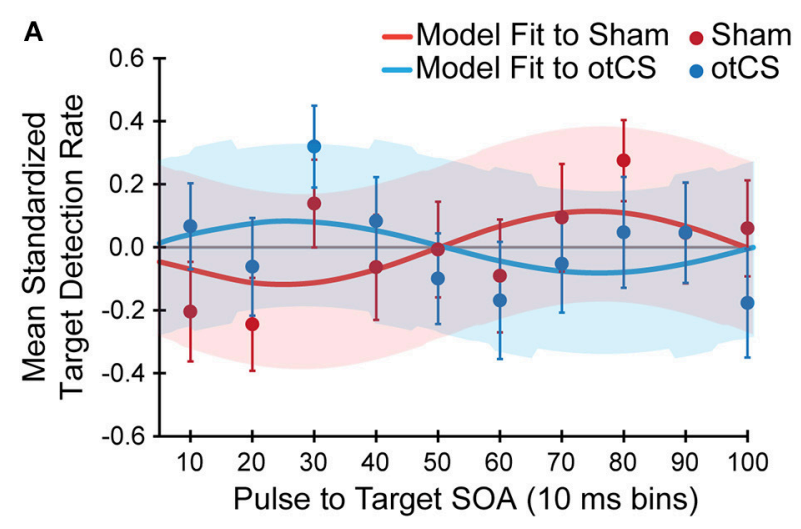

B

C

Sham
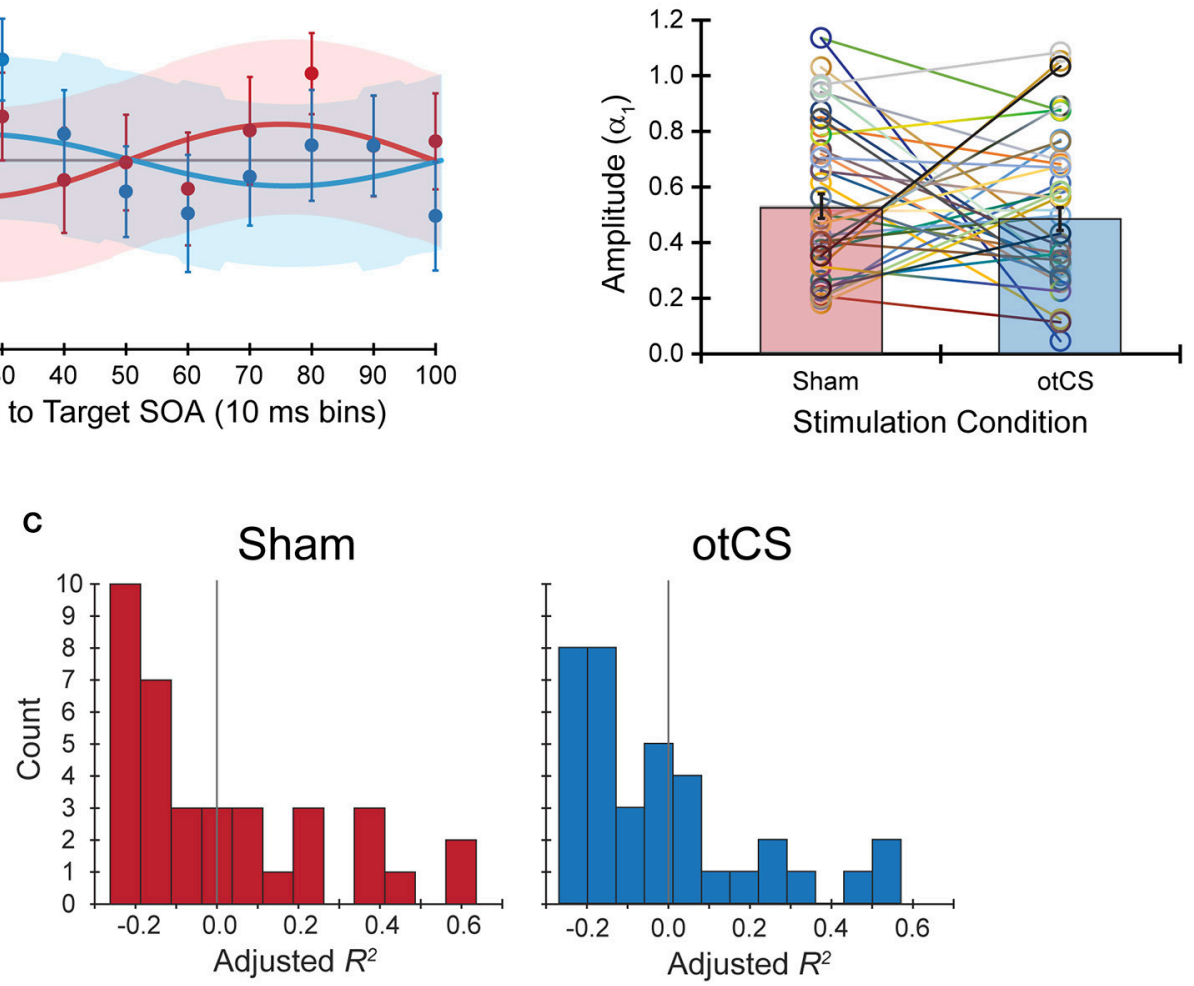

Stimulation Condition

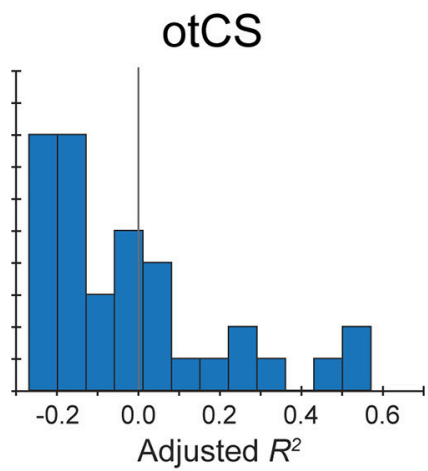

FIGURE 4 | (A) The mean standardized target detection rates in each $10 \mathrm{~ms}$ bin for the sham and otCS stimulation conditions overlaid by each fitted sine functions for the sham and cathodal otCS stimulation conditions. Error bars and shaded color regions indicate the SE of the mean standardized detection rates and model fits, respectively. (B) The open circles denote the individual amplitude $\left(\alpha_{1}\right)$ estimates for each participant in the sham and otCS conditions. Lines connect the data points from the same participant. The red and blue bars are the group averages in the sham and otCS conditions, respectively. The error bars are the SE. (C) Histograms of the goodness-of-fit measure, adjusted $R^{2}$, of the sinusoidal model to the mean standardized target detection rates in sham (left) and otCS (right) stimulation conditions. The larger the adjusted $r$-square value, the more variability in the detection rates explained by the model. Gray line marks a value of zero.

condition, stimulation condition order, and the stimulation condition and stimulation condition order interaction. The Bayes factor for inclusion (based on Bayesian model averaging; see Etz and Wagenmakers, 2017) indicates that the evidence most strongly supports the inclusion of the interaction term $\left(\mathrm{BF}_{\text {inclusion }}=604540.58\right)$, followed by stimulation condition 
order $\left(\mathrm{BF}_{\text {inclusion }}=146799.08\right)$, and then stimulation condition $\left(\mathrm{BF}_{\text {inclusion }}=107775.23\right)$. These results provided further evidence for a sequence effect, and indicate that the effect of stimulation condition should be looked at without the sequence effect confound.

To compensate for this sequence effects, target detection rates were normalized for each participant in each condition separately and were tested again with the same ANOVA. The statistical test also yielded no significant main effects or interactions including the interaction between stimulation condition and stimulation condition order (Figure 3B). There were no main effects or interactions with a $p$-value $<0.20$. In support of this conclusion, the accompanying Bayes factors indicate that the evidence is strongly in favor of the absence of any effects or interactions (all alternative models have a $\mathrm{BF}_{10}<0.05$ ).

Contrary to our hypothesis, the sinusoidal pattern of the target detection rates did not seem to be strongly modulated by the cathodal otCS stimulation pulses compared to the sham (Figure 4A). This is supported by a paired $t$-test which indicates that there is no significant difference in the estimated amplitude parameters $\left(\alpha_{1}\right)$ from the fitted sine functions to the otCS and sham behavioral data $\left[t_{(35)}=0.65, p=0.52\right.$; Figure 4B], and a Bayesian paired $t$-test showing that the data is 4.59 times better explained by the null hypothesis than the alternative $\left(\mathrm{BF}_{10}=0.22\right)$. Furthermore, a Wilcoxon signedranks test indicates that the amount of variability in the target detection rates accounted for by the sinusoidal model (adjusted $R^{2}$ value) did not differ significantly between the sham and otCS stimulation conditions $(Z=-0.58, p=0.56$; Figure 4C). A Bayesian Wilcoxon signed-ranks test provided support for the null hypothesis $\left[\mathrm{P}\left(\mathrm{H}_{0} \mid\right.\right.$ Data $\left.)=0.72\right]$.

Finally, the mean target detection rates across each experimental condition was examined to see if there was an effect of the otCS stimulation over the course of the trials. Mauchly's test indicated that the assumption of sphericity was violated for the stimulation condition $\mathrm{x}$ bins interaction, $W=0.065, p<0.01, \varepsilon=0.66$. The degrees of freedom were corrected using Greenhouse-Geisser estimates of sphericity. There was a significant main of bin on target detection rates $\left[F_{(11,385)}=4.78, p<0.001\right]$. The accompanying Bayes factor shows that the data are 25569.69 times more likely under the model including an effect for time course across consecutive trials. There was no significant main effect of stimulation condition $\left[F_{(1,35)}<1.00\right]$, nor a significant interaction between stimulation condition $\mathrm{x}$ bins $\left[F_{(7.23,253.19)}=0.65, p=0.72\right]$. As can be seen in Figure 5, there was a change in target detection rates across the duration of the task, but this change was about the same in both conditions. A post-hoc test using the Holm procedure to control for Type I errors revealed that the first 32 trials (bin $1 ; M=0.57, S E=0.03$ ) had significantly better target detection rates than the set of trials in bin $4(M=0.46$, $S E=0.03)$, bin $7(M=0.47, S E=0.02)$, and bin $8(M=0.48$, $S E=0.02)$. Because participants performed the task in three blocks of 128 trials, the end of the first block corresponds to bin 4 and the end of the second block corresponds to bin 8 . Therefore, the most likely explanation for these results is that the participants got fatigued toward the end of each block.

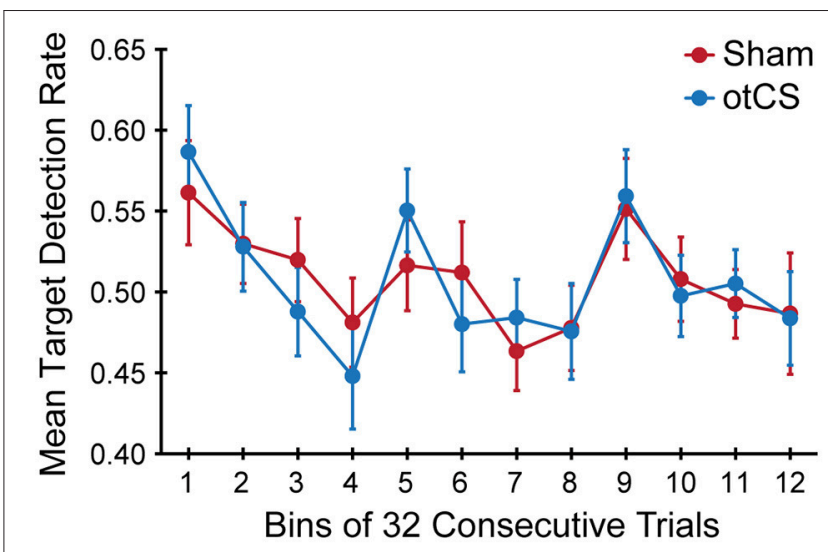

FIGURE 5 | Mean target detection rates rate in each bin of 32 consecutive trials across the three experimental blocks during the sham and otCS stimulation conditions. Error bars indicate the SE.

\section{EEG Data}

Although EEG data was recorded throughout the final three conditions, we were unable to analyze the data due to the presence of small random changes of stimulation intensity introduced by the stimulating device. Examples of this variability can be seen for two subjects in Figure 6.

\section{DISCUSSION}

The current studied aimed to provide a proof of principle that the entrainment of ongoing neural oscillations by rhythmic visual stimulation can be replicated with cathodal otCS at the same frequency. To this end, we attempted to modulate the phase of alpha oscillations in the posterior parietal cortex during a wellestablished visual detection task. Contrary to our hypothesis, there was no evidence that cathodal otCS stimulation pulses modulated target detection rates. We found that mean target detection rates during the otCS stimulation did not change as compared to sham stimulation. Furthermore, the sinusoidal pattern of the target detection rates did not seem to be strongly modulated by the cathodal otCS stimulation pulses compared to the sham. Together, these results did not provide significant evidence for $10 \mathrm{~Hz}$ cathodal otCS directly inducing modulation of alpha oscillations that can influence visual perception in a target detection task.

To the best of our analysis, cathodal otCS stimulation was not observed to modulate alpha oscillations and subsequent target detection rates. A major limitation of this study is that the efficacy of cathodal otCS can only by inferred from the perceptual and behavioral consequences of electrical stimulation during the target detection task. Although EEG was recorded throughout the experiment, we were not able to remove the otCS-induced artifacts. As a result, we have no direct electrophysiological evidence that the cathodal otCS stimulation interacted with the ongoing brain oscillations. Therefore, we cannot eliminate technical or methodological issues as the explanation for a lack of measurable behavior effects. For example, it is possible 

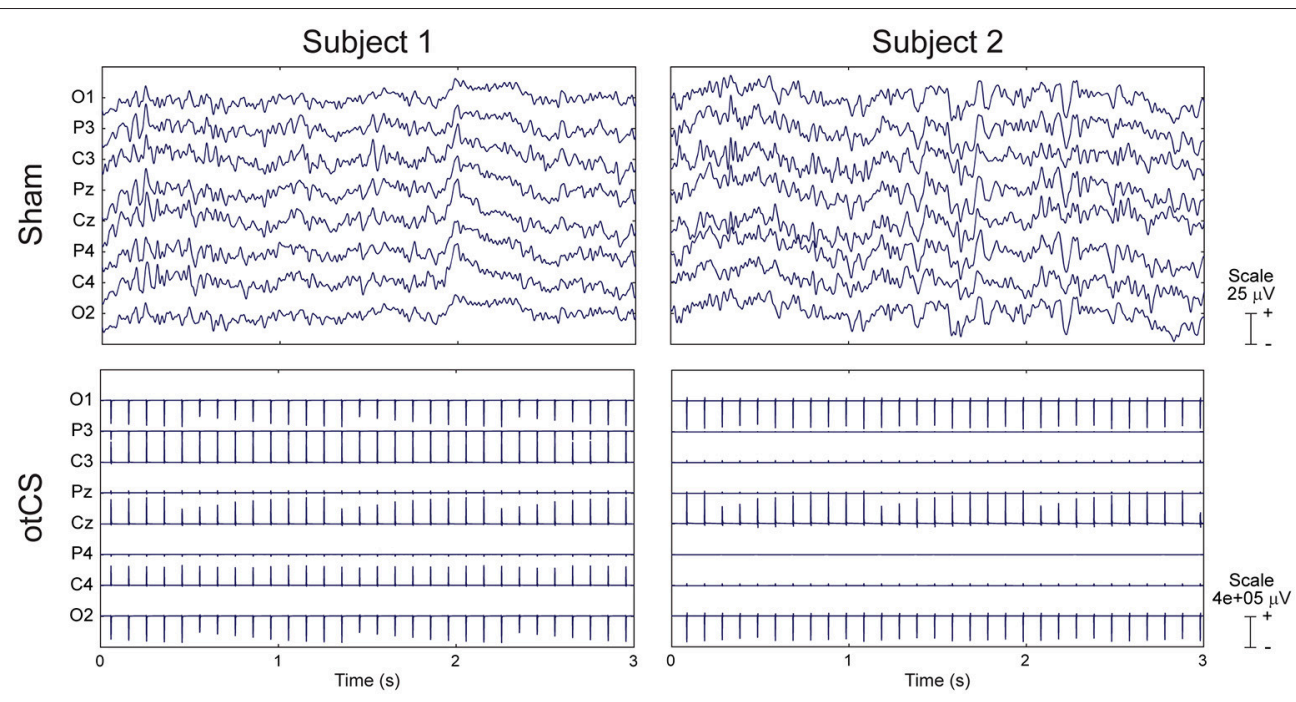

FIGURE 6 | Raw EEG data from two subjects during the sham condition (Top) and otCS condition (Bottom). Note the between- and within-subject differences in the otCS induced artifacts. In particular, the non-linear variability of the stimulation artifact within each electrode across time.

that the stimulation intensity or duration was not sufficient for inducing modulation of endogenous alpha oscillation. However, it is unlikely that stimulation intensity was too low to induce effects because previous studies have used similar intensities with observable effects (Moliadze et al., 2012; Neuling et al., 2015). Notably, Ruhnau et al. (2016) found that even a very low tACS current $(0.05 \mathrm{~mA})$ can induce patterns of entrainment similar to higher intensities. Even so, future studies might stimulate at higher intensities and include a control condition using a different frequency to definitively eliminate this possibility. Insufficient stimulation duration is also an unlikely explanation because there was no change in target detection rates compared to sham over course of the target detection task (see Figure 5). Furthermore, the three blocks of the target detection task took at least 10 mins which is considered enough time to induce effects in the ongoing oscillations (Antal et al., 2008; Thair et al., 2017).

It is also possible that using a $10 \mathrm{~Hz}$ stimulation frequency for all participants rather than matching the otCS frequency to each individuals' peak alpha frequency reduced the efficacy of cathodal otCS. Several lines of evidence have shown that effective modulation of endogenous oscillations by periodic brain stimulation depends on matching the stimulation frequency to the rhythmic activity. For example, a study using optogenetic stimulation and multichannel slice electrophysiology found that a weak sine-wave electric field can enhance ongoing oscillatory activity, but only when the stimulation frequency was matched to the endogenous oscillation (Schmidt et al., 2014). Furthermore, a meta-analysis of 51 sham controlled experiments that investigated the effects of tACS on perception and cognitive performance, Schutter and Wischnewski (2016) found that performance is more likely to increase when tACS is administered based on individual spectral information. Together, these results suggest that the efficacy of cathodal otCS in the current study might have been greatly reduced because we did not control for inter-individual differences of endogenous alpha oscillations. However, using a $10 \mathrm{~Hz}$ stimulation frequency rather than matching the otCS frequency to individual peak frequencies might not have been as important a factor as it might seem. Specifically, even in the same participant, individual endogenous oscillatory activity varies during the course of a given task which could decrease the effects of stimulation even when the individual peak frequency was applied (Woods et al., 2016).

Another factor that could have reduced the efficacy of this method was that we did not control the timing of the otCS stimulation with regards to the target detection task. As a result, state-dependent differences in cortical activity across individuals prior to otCS may influence the effects of subsequent stimulation, introducing a possible source of variability (Silvanto and PascualLeone, 2008). However, this is an unlikely explanation because much of the variability due to differences across individuals would have been accounted for in the sham condition and by blocking on participants in the statistical analysis. Therefore, it is unlikely that state-dependent differences in cortical activity could significantly contribute to the lack of behavioral differences between the otCS and sham conditions in the target detection task.

In addition to the technical and methodological limitations mentioned above, individual differences in the brain's susceptibility to otCS is another factor that may contribute to the lack of an observable effect. Anatomical variation including scalp-brain distance, gyral folding of the cerebral cortex, and thickness of corticospinal fluid layer and skull can have a significant impact on the effects of transcranial current stimulation (Nitsche et al., 2008; Opitz et al., 2015). In the future, computational models might help overcome these inter-individual differences by allowing researchers to select the optimal set of stimulation parameters for each individual based on models of current flow (Truong et al., 2014; Dmochowski 
et al., 2017). However, the fact that tCS can cause non-linear effects at the neurophysiological and behavioral level (Bonaiuto, 2015), and the difficulty in obtaining data in vivo to validate the computational models (Bai et al., 2013), necessitate further model refinements and development. Furthermore, adjusting stimulation frequency as a function of intrinsic brain oscillations properties could also be more effective at modulating perception.

The results of the current study suggest that $10-\mathrm{Hz}$ cathodal otCS stimulation does not directly induce modulation of alpha oscillations that can influence visual perception in a target detection task. Part of this null result might be explained by individual differences in peak alpha frequency, statedependent changes in cortical activity, and susceptibility to otCS stimulation. However, technical and methodological issues might also contribute a lack of observable differences in visual perception. In the absence of electrophysiological evidence, it is important to be cautious about forming any firm conclusions based on the current study. Further research is needed to convincingly eliminate cathodal otCS stimulation as a means of modulating endogenous alpha oscillations in the posterior

\section{REFERENCES}

Alekseichuk, I., Diers, K., Paulus, W., and Antal, A. (2016). Transcranial electrical stimulation of the occipital cortex during visual perception modifies the magnitude of BOLD activity: a combined tES-fMRI approach. Neuroimage 140, 110-117. doi: 10.1016/j.neuroimage.2015.11.034

Ali, M. M., Sellers, K. K., and Fröhlich, F. (2013). Transcranial alternating current stimulation modulates large-scale cortical network activity by network resonance. J. Neurosci. 33, 11262-11275. doi: 10.1523/JNEUROSCI.5867-12.2013

Antal, A., and Paulus, W. (2008). Transcranial direct current stimulation and visual perception. Perception 37, 367-374. doi: 10.1068/p5872

Antal, A., and Paulus, W. (2013). Transcranial alternating current stimulation (tACS). Front. Hum. Neurosci. 7:317. doi: 10.3389/fnhum.2013.00317

Antal, A., Boros, K., Poreisz, C., Chaieb, L., Terney, D., and Paulus, W. (2008). Comparatively weak after-effects of transcranial alternating current stimulation (tACS) on cortical excitability in humans. Brain Stimul. 1, 97-105. doi: 10.1016/j.brs.2007.10.001

Antal, A., Kincses, T. Z., Nitsche, M. A., Bartfai, O., and Paulus, W. (2004a). Excitability changes induced in the human primary visual cortex by transcranial direct current stimulation: direct electrophysiological evidence. Investig. Opthalmol. Vis. Sci. 45, 702-707. doi: 10.1167/iovs.03-0688

Antal, A., Nitsche, M. A., Kruse, W., Kincses, T. Z., Hoffmann, K. P., and Paulus, W. (2004b). Direct current stimulation over V5 enhances visuomotor coordination by improving motion perception in humans. J. Cogn. Neurosci. 16, 521-527. doi: 10.1162/089892904323057263

Antonenko, D., Faxel, M., Grittner, U., Lavidor, M., and Flöel, A. (2016). Effects of transcranial alternating current stimulation on cognitive functions in healthy young and older adults. Neural Plast. 2016:4274127. doi: 10.1155/2016/4274127

Bai, S., Loo, C., and Dokos, S. (2013). A review of computational models of transcranial electrical stimulation. Crit. Rev. Biomed. Eng. 41, 21-35. doi: 10.1615/CritRevBiomedEng.2013007163

Benavoli, A., Mangili, F., Corani, G., Zaffalon, M., and Ruggeri, F. (2014). "A bayesian wilcoxon signed-rank test based on the dirichlet process," in Proceedings of the 31st International Conference on Machine Learning (Beijing). Available online at: http://ipg.idsia.ch/preprints/benavoli2014a.pdf

Bonaiuto, J. J. (2015). "Chapter 4: Understanding the nonlinear physiological and behavioral effects of tDCS through computational neurostimulation," in Progress in Brain Research, ed S. Bestmann (Amsterdam: Elsevier), 75-103.

Brainard, D. H. (1997). The psychophysics toolbox. Spat. Vis. 10, 433-436. doi: $10.1163 / 156856897 \mathrm{X} 00357$ parietal area. However, the current study provides the first evidence supporting that conclusion.

\section{AUTHOR CONTRIBUTIONS}

SS and KM contributed conception and design of the study. SS performed the statistical analysis. Both authors interpreted the data and wrote the article. Both authors approved the final version of the manuscript.

\section{FUNDING}

This work was supported by a Natural Science and Engineering Research Council of Canada (NSERC) discovery grant (\#04792) to KM.

\section{ACKNOWLEDGMENTS}

The authors would like to thank Anna Kim for assistance with data collection.

Brignani, D., Ruzzoli, M., Mauri, P., and Miniussi, C. (2013). Is transcranial alternating current stimulation effective in modulating brain oscillations? PLoS ONE 8:e56589. doi: 10.1371/journal.pone.0056589

Brunoni, A. R., Amadera, J., Berbel, B., Volz, M. S., Rizzerio, B. G., and Fregni, F. (2011). A systematic review on reporting and assessment of adverse effects associated with transcranial direct current stimulation. Int. J. Neuropsychopharmacol. 14, 1133-1145. doi: 10.1017/S1461145710001690

Busch, N. A., Dubois, J., and VanRullen, R. (2009). The phase of ongoing EEG oscillations predicts visual perception. J. Neurosci. 29, 7869-7876. doi: 10.1523/JNEUROSCI.0113-09.2009

de Graaf, T. A., Gross, J., Paterson, G., Rusch, T., Sack, A. T., and Thut, G. (2013). Alpha-band rhythms in visual task performance: phase-locking by rhythmic sensory stimulation. PLoS ONE 8:e60035. doi: 10.1371/journal.pone.00 60035

Delorme, A., and Makeig, S. (2004). EEGLAB: an open source toolbox for analysis of single-trial EEG dynamics including independent component analysis. J. Neurosci. Methods 134, 9-21. doi: 10.1016/j.jneumeth.2003.10.009

Dmochowski, J. P., Koessler, L., Norcia, A. M., Bikson, M., and Parra, L. C. (2017). Optimal use of EEG recordings to target active brain areas with transcranial electrical stimulation. Neuroimage 157, 69-80. doi: 10.1016/j.neuroimage.2017.05.059

Etz, A., and Wagenmakers, E.-J. (2017). JBS Haldane's contribution to the Bayes factor hypothesis test. Stat. Sci. 32, 313-329. doi: 10.1214/16-STS599

Fröhlich, F. (2015). "Chapter 3: Experiments and models of cortical oscillations as a target for noninvasive brain stimulation," in Progress in Brain Research, ed S. Bestmann, (Amsterdam: Elsevier), 41-73.

Fröhlich, F., Sellers, K. K., and Cordle, A. L. (2015). Targeting the neurophysiology of cognitive systems with transcranial alternating current stimulation. Expert Rev. Neurother. 15, 145-167. doi: 10.1586/14737175.2015.992782

García-Pérez, M. A. (1998). Forced-choice staircases with fixed step sizes: asymptotic and small-sample properties. Vision Res. 38, 1861-1881. doi: 10.1016/S0042-6989(97)00340-4

Gratton, G., Coles, M. G., and Donchin, E. (1983). A new method for off-line removal of ocular artifact. Electroencephalogr. Clin. Neurophysiol. 55, 468-484. doi: 10.1016/0013-4694(83)90135-9

Gulbinaite, R., van Viegen, T., Wieling, M., Cohen, M. X., and VanRullen, R. (2017). Individual alpha peak frequency predicts $10 \mathrm{~Hz}$ flicker effects on selective attention. J. Neurosci. 37, 10173-10184. doi: 10.1523/JNEUROSCI.1163-17.2017

Haegens, S., Nácher, V., Luna, R., Romo, R., and Jensen, O. (2011). $\alpha$-Oscillations in the monkey sensorimotor network influence discrimination performance by 
rhythmical inhibition of neuronal spiking. Proc. Natl. Acad. Sci. U.S.A. 108, 19377-19382. doi: 10.1073/pnas.1117190108

Helfrich, R. F., Schneider, T. R., Rach, S., Trautmann-Lengsfeld, S. A., Engel, A. K., and Herrmann, C. S. (2014). Entrainment of brain oscillations by transcranial alternating current stimulation. Curr. Biol. 24, 333-339. doi: 10.1016/j.cub.2013.12.041

Herrmann, C. S., Rach, S., Neuling, T., and Strüber, D. (2013). Transcranial alternating current stimulation: a review of the underlying mechanisms and modulation of cognitive processes. Front. Hum. Neurosci. 7:279. doi: $10.3389 /$ fnhum.2013.00279

Horvath, J. C., Forte, J. D., and Carter, O. (2015). Evidence that transcranial direct current stimulation (tDCS) generates little-to-no reliable neurophysiologic effect beyond MEP amplitude modulation in healthy human subjects: a systematic review. Neuropsychologia 66, 213-236. doi: 10.1016/j.neuropsychologia.2014.11.021

Jaberzadeh, S., Bastani, A., and Zoghi, M. (2014). Anodal transcranial pulsed current stimulation: a novel technique to enhance corticospinal excitability. Clin. Neurophysiol. 125, 344-351. doi: 10.1016/j.clinph.2013.08.025

Jackson, M. P., Rahman, A., Lafon, B., Kronberg, G., Ling, D., Parra, L. C., et al. (2016). Animal models of transcranial direct current stimulation: methods and mechanisms. Clin. Neurophysiol. 127, 3425-3454. doi: 10.1016/j.clinph.2016.08.016

Jarosz, A. F., and Wiley, J. (2014). What are the odds? a practical guide to computing and reporting bayes factors. J. Probl. Solving 7. doi: $10.7771 / 1932-6246.1167$

JASP Team (2018). JASP. Amsterdam.

Keitel, C., Quigley, C., and Ruhnau, P. (2014). Stimulus-driven brain oscillations in the alpha range: entrainment of intrinsic rhythms or frequency-following response? J. Neurosci. 34, 10137-10140. doi: 10.1523/JNEUROSCI.1904-14.2014

Kingdom, F. A. A., and Prins, N. (2016). "Chapter 5: Adaptive methods," in Psychophysics (San Diego, CA: Academic Press), 119-148.

Kizuk, S. A. D., and Mathewson, K. E. (2017). Power and phase of alpha oscillations reveal an interaction between spatial and temporal visual attention. J. Cogn. Neurosci. 29, 480-494. doi: 10.1162/jocn_a_01058

Klimesch, W. (2012). Alpha-band oscillations, attention, and controlled access to stored information. Trends Cogn. Sci. 16, 606-617. doi: 10.1016/j.tics.2012.10.007

Klimesch, W., Sauseng, P., and Hanslmayr, S. (2007). EEG alpha oscillations: the inhibition-timing hypothesis. Brain Res. Rev. 53, 63-88. doi: 10.1016/j.brainresrev.2006.06.003

Kohli, S., and Casson, A. J. (2015). "Removal of transcranial a.c. current stimulation artifact from simultaneous EEG recordings by superposition of moving averages," in 2015 37th Annual International Conference of the IEEE Engineering in Medicine and Biology Society (EMBC) (Milan: IEEE), 3436-3439.

Lafon, B., Henin, S., Huang, Y., Friedman, D., Melloni, L., Thesen, T., et al. (2017). Low frequency transcranial electrical stimulation does not entrain sleep rhythms measured by human intracranial recordings. Nat. Commun. 8:1199. doi: 10.1038/s41467-01701045-x

Laufs, H., Kleinschmidt, A., Beyerle, A., Eger, E., Salek-Haddadi, A., Preibisch, C., et al. (2003). EEG-correlated fMRI of human alpha activity. Neuroimage 19, 1463-1476. doi: 10.1016/S1053-8119(03)00286-6

Lawrence, M. A. (2016). ez: Easy Analysis and Visualization of Factorial Experiment. Available online at: https://cran.r-project.org/package $=\mathrm{ez}$

Liu, Z., de Zwart, J. A., van Gelderen, P., Kuo, L. W., and Duyn, J. H. (2012). Statistical feature extraction for artifact removal from concurrent fMRI-EEG recordings. Neuroimage 59, 2073-2087. doi: 10.1016/j.neuroimage.2011.10.042

Marshall, L., Helgadóttir, H., Mölle, M., and Born, J. (2006). Boosting slow oscillations during sleep potentiates memory. Nature 444, 610-613. doi: $10.1038 /$ nature 05278

Marshall, T. R., Esterer, S., Herring, J. D., Bergmann, T. O., and Jensen, O. (2016). On the relationship between cortical excitability and visual oscillatory responses - a concurrent tDCS-MEG study. Neuroimage 140, 41-49. doi: 10.1016/j.neuroimage.2015.09.069

Mathewson, K. E., Beck, D. M., Ro, T., Maclin, E. L., Low, K. A., Fabiani, M., et al. (2014). Dynamics of alpha control: preparatory suppression of posterior alpha oscillations by frontal modulators revealed with combined EEG and Event-related Optical Signal. J. Cogn. Neurosci. 26, 2400-2415. doi: $10.1162 /$ jocn_a_00637

Mathewson, K. E., Gratton, G., Fabiani, M., Beck, D. M., and Ro, T. (2009). To see or not to see: prestimulus phase predicts visual awareness. J. Neurosci. 29, 2725-2732. doi: 10.1523/JNEUROSCI.3963-08.2009

Mathewson, K. E., Lleras, A., Beck, D. M., Fabiani, M., Ro, T., and Gratton, G. (2011). Pulsed out of awareness: EEG alpha oscillations represent a pulsed-inhibition of ongoing cortical processing. Front. Psychol. 2:99. doi: 10.3389/fpsyg.2011.00099

Moliadze, V., Atalay, D., Antal, A., and Paulus, W. (2012). Close to threshold transcranial electrical stimulation preferentially activates inhibitory networks before switching to excitation with higher intensities. Brain Stimul. 5, 505-511. doi: 10.1016/j.brs.2011.11.004

Neuling, T., Rach, S., Wagner, S., Wolters, C. H., and Herrmann, C. S. (2012a). Good vibrations: oscillatory phase shapes perception. Neuroimage 63, 771-778. doi: 10.1016/j.neuroimage.2012.07.024

Neuling, T., Ruhnau, P., Fuscà, M., Demarchi, G., Herrmann, C. S., and Weisz, N. (2015). Friends, not foes: magnetoencephalography as a tool to uncover brain dynamics during transcranial alternating current stimulation. Neuroimage 118, 406-413. doi: 10.1016/j.neuroimage.2015.06.026

Neuling, T., Wagner, S., Wolters, C. H., Zaehle, T., and Herrmann, C. S. (2012b). Finite-element model predicts current density distribution for clinical applications of tDCS and tACS. Front. Psychiatry 3:83. doi: 10.3389/fpsyt.2012.00083

Nitsche, M. A., Cohen, L. G., Wassermann, E. M., Priori, A., Lang, N., Antal, A., et al. (2008). Transcranial direct current stimulation: state of the art 2008. Brain Stimul. 1, 206-223. doi: 10.1016/j.brs.2008.06.004

Notbohm, A., Kurths, J., and Herrmann, C. S. (2016). Modification of brain oscillations via rhythmic light stimulation provides evidence for entrainment but not for superposition of event-related responses. Front. Hum. Neurosci. 10:10. doi: 10.3389/fnhum.2016.00010

Opitz, A., Paulus, W., Will, S., Antunes, A., and Thielscher, A. (2015). Determinants of the electric field during transcranial direct current stimulation. Neuroimage 109, 140-150. doi: 10.1016/j.neuroimage.2015.01.033

Paulus, W. (2011). Transcranial electrical stimulation (tES - tDCS; tRNS, tACS) methods. Neuropsychol. Rehabil. 21, 602-617. doi: 10.1080/09602011.2011.557292

Paulus, W., Nitsche, M. A., and Antal, A. (2016). Application of transcranial electric stimulation (tDCS, tACS, tRNS). Eur. Psychol. 21, 4-14. doi: 10.1027/1016-9040/a000242

Pelli, D. G. (1997). The videotoolbox software for visual psychophysics: transforming numbers into movies. Spat. Vis. 10, 437-442. doi: 10.1163/156856897X00366

Polanía, R., Nitsche, M. A., and Ruff, C. C. (2018). Studying and modifying brain function with non-invasive brain stimulation. Nat. Neurosci. 21, 174-187. doi: 10.1038/s41593-017-0054-4

R Core Team (2013). R: A Language and Environment for Statistical Computing. Vienna.

Reato, D., Rahman, A., Bikson, M., and Parra, L. C. (2013). Effects of weak transcranial alternating current stimulation on brain activity-a review of known mechanisms from animal studies. Front. Hum. Neurosci. 7:687. doi: 10.3389/fnhum.2013.00687

Ruhnau, P., Neuling, T., Fuscá, M., Herrmann, C. S., Demarchi, G., Weisz, N., et al. (2016). Eyes wide shut: transcranial alternating current stimulation drives alpha rhythm in a state dependent manner. Sci. Rep. 6:27138. doi: 10.1038/srep27138

Schmidt, S. L., Iyengar, A. K., Foulser, A. A., Boyle, M. R., and Fröhlich, F. (2014). Endogenous cortical oscillations constrain neuromodulation by weak electric fields. Brain Stimul. 7, 878-889. doi: 10.1016/j.brs.2014. 07.033

Schutter, D. J., and Wischnewski, M. (2016). A meta-analytic study of exogenous oscillatory electric potentials in neuroenhancement. Neuropsychologia $\quad 86, \quad 110-118$. doi: 10.1016/j.neuropsychologia.2016. 04.011

Siever, D. (2014). Two Earclips, Wires, a Little Box and Presto - The Wonderful World of Cranio-Electro Stimulation. Available online at: https://mindalive. com/default/assets/File/CES\%20Article\%20-\%20Jan\%2026-2015.pdf 
Silvanto, J., and Pascual-Leone, A. (2008). State-dependency of transcranial magnetic stimulation. Brain Topogr. 21, 1-10. doi: 10.1007/s10548-008-0067-0

Simonsmeier, B. A., and Grabner, R. H. (2017). Electrical brain stimulation (tES) improves learning more than performance: a meta-analysis. Neurosci. Biobehav. Rev. 84, 171-181. doi: 10.1016/j.neubiorev.2017. 11.001

Spaak, E., de Lange, F. P., and Jensen, O. (2014). Local entrainment of alpha oscillations by visual stimuli causes cyclic modulation of perception. J. Neurosci. 34, 3536-3544. doi: 10.1523/JNEUROSCI.4385-13.2014

Thair, H., Holloway, A. L., Newport, R., and Smith, A. D. (2017). Transcranial direct current stimulation (tDCS): a beginner's guide for design and implementation. Front. Neurosci. 11:641. doi: 10.3389/fnins.2017.00641

Tremblay, S., Lepage, J. F., Latulipe-Loiselle, A., Fregni, F., Pascual-Leone, A., and Théoret, H. (2014). The uncertain outcome of prefrontal tDCS. Brain Stimul. 7, 773-783. doi: 10.1016/j.brs.2014.10.003

Truong, D., Minhas, P., Nair, A., and Bikson, M. (2014). “Computational modeling assisted design of optimized and individualized transcranial direct current stimulation protocols," in The Stimulated Brain ed R. C. Kadosh, (San Diego, CA: Elsevier; Academic Press), 85-115.

Veniero, D., Benwell, C. S. Y., Ahrens, M. M., and Thut, G. (2017). Inconsistent effects of parietal $\alpha$-tACS on pseudoneglect across two experiments: a failed internal replication. Front. Psychol. 8:952. doi: 10.3389/fpsyg.2017.00952

Vosskuhl, J., Strüber, D., and Herrmann, C. S. (2015). Transcranial alternating current stimulation: entrainment and function control of neuronal networks. Nervenarzt 86, 1516-1522. doi: 10.1007/s00115-015-4317-6
Wagenmakers, E. J., Marsman, M., Jamil, T., Ly, A., Verhagen, J., Love, J., et al. (2017). Bayesian inference for psychology. Part I: theoretical advantages and practical ramifications. Psychon. Bull. Rev. 24, 1-23. doi: $10.3758 / s 13423-017-1343-3$

Woods, A. J., Antal, A., Bikson, M., Boggio, P. S., Brunoni, A. R., Celnik, P., et al. (2016). A technical guide to tDCS, and related non-invasive brain stimulation tools. Clin. Neurophysiol. 127, 1031-1048. doi: 10.1016/j.clinph.2015.11.012

Zaehle, T., Sandmann, P., Thorne, J. D., Jäncke, L., Herrmann, C. S., Engel, A., et al. (2011). Transcranial direct current stimulation of the prefrontal cortex modulates working memory performance: combined behavioural and electrophysiological evidence. BMC Neurosci. 12:2. doi: 10.1186/1471-2202-12-2

Conflict of Interest Statement: The authors declare that the research was conducted in the absence of any commercial or financial relationships that could be construed as a potential conflict of interest.

The reviewer CK and handling Editor declared their shared affiliation.

Copyright $(02018$ Sheldon and Mathewson. This is an open-access article distributed under the terms of the Creative Commons Attribution License (CC BY). The use, distribution or reproduction in other forums is permitted, provided the original author(s) and the copyright owner are credited and that the original publication in this journal is cited, in accordance with accepted academic practice. No use, distribution or reproduction is permitted which does not comply with these terms. 